\title{
Exploration and Practice of Curriculum Teaching Reform of Communication Network Management
}

\author{
Jiang Haixia*, Tong Wei, Zhou Xingyu, Zheng Xiang, Zhu Lei \\ College of Communication Engineering, PLA University of Science and Technology, Nanjing, China \\ Email address: \\ jhx123@139.com (Jiang Haixia) \\ ${ }^{*}$ Corresponding author
}

To cite this article:

Jiang Haixia, Tong Wei, Zhou Xingyu, Zheng Xiang, Zhu Lei. Exploration and Practice of Curriculum Teaching Reform of Communication Network Management. Science Innovation. Vol. 5, No. 1, 2017, pp. 57-62. doi: 10.11648/j.si.20170501.20

Received: December 28, 2016; Accepted: March 6, 2017; Published: March 15, 2017

\begin{abstract}
A significant research on reform and practice in teaching for the course of Communication Network Management is presented in this paper.The purpose of this curriculum reform is to solve the problems existing in the characteristics of the course itself, the characteristics of teaching objects and the traditional teaching methods. The specific course teaching model is gradually built up through optimizing teaching content system, reforming teaching process, such as theoretical teaching, experimental teaching and assessment methods. This novel teaching model explores the theoretical teaching model based on PoPBL, the experimental teaching model based on CDIO and the application of a variety of modern teaching methods, and adopts a scientific and effective teaching effect evaluation mechanism. It is proved by the practice that the teaching quality and comprehensive ability of undergraduates are being improved. These improvements indicate that the reform on the course is effective as the goal of course planning is successfully achieved.
\end{abstract}

Keywords: Teaching Reform, Communication Network Management, Experiment Teaching, CDIO

\section{“通信网管理”课程教学改革探索与实践}

蒋海霞, 童玮, 周星宇, 郑翔, 朱磊

解放军理工大学通信工程学院, 南京, 中国

邮箱

jhx123@139. com（蒋海霞）

摘要：针对 “通信网管理” 课程自身的特点、授课对象的特点以及传统教学方法中存在的问题, 从课程的理论教学、 实验教学、考核方式以及教学手段等环节上进行改革, 优化课程教学内容体系, 探索与实践了基于PoPBL的理论教学模 式、基于CDI0的工程实验教学模式, 结合多种现代化教学手段的运用, 采用了科学、有效的教学效果评价机制, 教学 实践表明, 对通信网管理课程教学改革的探索与实践, 激发了学生学习兴趣与学习动力, 在培养和提高学生工程实践 能力的同时, 达到了提升教学效果的目的。

关键词: 教学改革, 通信网管理, 实验教学, CDI0 


\section{1. 引言}

通信网管理是指与通信网络的运营、管理、维护和供 应相关的活动、方法、规程以及工具 [1]。该门课程具有 知识覆盖面广、知识点散乱、相关知识多等特点, 教学探 索性较强, 因此在教学中我们遵循重基础、学以致用和理 论联系实际的指导思想, 贯彻把复杂的网络管理理论与当 前主流的网络管理实际问题相结合的理念, 注重培养学生 分析问题、解决问题的能力, 提高学生网络管理技能 [2]。

该课程是我校通信兵指挥专业大专班的专业必修课, 与本科学生的教学不同, 由于大专班学生的学习动力普遍 不足, 文化基础比较薄弱, 加上课程自身的特点等多方面 的因素, 导致课程的教学效果欠佳, 因此针对这门课程自 身的特点以及授课对象的特点, 在授课过程中我们尝试从 教法以及学法方面做了一些改革探索, 主要目的就是要激 发学生的学习兴趣, 提升课程的教学效果。

\section{2. 传统教学方法中存在问题与原因探寻}

\section{1. 存在的问题}

通信网管理学习起来并不容易, 它是一门交叉学科, 涉及到了网络可靠性理论、网络优化理论、人工智能理论、 面向对象的分析与设计、数据库技术及计算机仿真技术等 多种知识, 具有相当的广度和深度。从课程教学实践来看, 自该课程开设以来, 教师感觉通信网管理课程的内容抽象 而且繁杂, 概念多、知识点多, 按照传统方式授课难以向 学生讲清楚, 而学生则往往是知其然不知其所以然, 可能 可以靠死记硬背的方式掌握一些内容, 如OSI网络管理模 型等, 但为什么要设计这样的管理模型却全然不知, 结果 就是教师教得累, 学生学得枯燥乏味, 学习时难以找到着 力点, 难以激发学习热情和兴趣, 学习效率较低, 教学效 果不理想 $[3]$ 。

\section{2. 原因探究}

在我校深入开展教法改革的大背景下, 在数年教学实 践的基础上, 我们深入研究分析了造成通信网管理教学效 果差的原因, 主要有以下三点: 一是传统教学过于重视理 论讲授却忽视与实践结合, 理论与实践脱钩, 未能做到激 发学生的求知欲; 二是传统教学重点在于授人以鱼, 却未 做到授人以渔, 未能引导学生主动思考; 三是实践环节依 葫芦画㼼, 缺乏创新性与挑战性, 因此对学生缺乏吸引力。

针对上述问题, 根据原因探究, 我们从理论教学、实 践教学以及考核方法、教学手段等几个方面进行了教学上 的创新探索。

\section{3. 理论教学方法改革}

理论教学是实验教学的基础, 实验教学能加深学生对 理论知识的理解与掌握。因此理论教学与实验教学是相辅 相成的。在教学方法改革中, 两者并重, 同时根据理论教 学与实验教学这两个教学环节的特点, 我们进行了不同的 教学模式的改革。

\section{1. 理论教学基本方案}

虽然通信网管理的理论知识体系庞杂, 但根据人才培 养方案, 本门面向大专班开设的课程只有 10 学时的理论教 学时间。在现有规定教学时间情况下, 如果仍然按照系统 传授理论知识的思路展开教学, 势必让教师像赶火车一样 赶进度, 而学生由于基础知识本身就比较薄弱, 学习起来 更是云里雾里, 抓不住重点。具体理论教学内容与学时分 配如表1所示。

表1 理论教学内容与学时分配。

\begin{tabular}{lll}
\hline 序号 & 教学内容 & 课堂教学学时 \\
\hline 1 & 网络管理功能与标准化 & 2 \\
2 & 业务量控制技术 & 2 \\
3 & 路由选择技术 & 2 \\
4 & 网络自愈技术 & 2 \\
5 & 网络信息安全技术 & 2 \\
总计 & & 10 \\
\hline
\end{tabular}

教学改革中, 我们将理论教学由传统的系统化教学向 专题化教学转变, 因此在实施中, 我们在研究PBL教学模 式的基础上, 采用了PoPBL的教学模式实施理论教学改革。 下面简单介绍具体实施方法与心得体会。

\subsection{PBL教学模式简介}

PBL即基于问题的学习 (Problem-based Learning), 简称PBL, 也称作问题式学习，是基于现实世界的问题为 中心、以学生为主体的教育与学习模式, 目前已成为国际 上较流行的一种教学方法。目前对于什么是PBL教学这个 问题, 不同的学者有不同的理解, 综合国内外对PBL模式 的研究和拓展, 可以将PBL归纳为三种模式: 基于问题式 学习模式 (Prolem-based Learning) 、基于项目式学习 模式 (Project-based Learning) 及以问题为导向、基于 项目学习模式 (Prolem-oriented and Project-based Learning)。

对PBL内涵的多样化理解源于PBL在实践中的应用领 域不同，在不同的教育文化背景下、不同学科领域中, PBL 在实践中已经呈现出不同的形态与模式, 向着多元化方向 发展, 虽然对于PBL的概念界定不同, 但无论是哪一种界 定, 均认为PBL是一种新的教学理念、教学模式和教学方 法。不论PBL在实践中采用何种形式, 它的概念内涵集中 体现在以下四个方面。

（1）学习方法: 以问题为导向、基于项目的学习方 法。让学生在解决现实问题中获取知识、学会学习, 并通 过反思构建自己的知识体系;

（2）学习内容: 跨越了传统的单一学科, 学生通过 解决具体问题, 可能需要多学科的知识, 学科交叉成为PBL 模式一大特征;

（3）学习形式: 强调以小组协作的形式, 通过相互 合作、相互交流来解决问题, 分享知识, 培养学生组织管 理、自主管理以及合作与交流的能力;

(4) 学习主体: 以学生为中心, 学生可以自己选题, 自己设置学习目标, 结合教学总目标根据兴趣点进行科学 
研究, 成为独立的思考者与学习者。而教师是作为一个合 作者和教练, 引导学习。

综上所述, 在工科专业课程教学中选用PBL教学模式, 有利于突出学生的主体地位, 激发学生自主学习的热情, 促进学生学习能力、实践能力、创新能力和合作能力的培 养 $[5]$ 。

\section{3. 基于PoPBL的理论教学}

在深入思考通信网管理课程特点的情况下, 我们采用 了以问题为导向、基于项目学习模式 (PoPBL) 这种以问 题为导向的鼓励式教学模式。

具体做法是由教师提出一个具体的建设项目, 结合这 个项目提出具体待解决的问题, 以这些问题为引导, 激发 学生思考和尝试解决这些问题, 最后对这些问题及学生的 解决方法进行总结归纳, 在此基础上提出教材上的思路和 方法, 请学生提炼出教材上通信网管理具体方法的特点, 从而实现了授之以渔的目的。显然, 这样能够充分发挥学 生的主体作用, 激发学生独立思考和创新意识。让学生带 着兴趣去主动学习, 能更好地培养学生发现问题、解决问 题以及开拓创新的能力。

针对课程本身特点以及结合以往教学实践和学生具 体情况, 在课程教学中, 我们通过采用 “任务驱动”、“案 例教学” 等多种教学模式实施理论教学改革, 把结合实际 的生动问题和校园网建设及管理的项目案例融入教学内 容中, 通过引导学生思考问题解决的方法, 进而掌握授课 内容的内涵, 引导学生构建通信网管理的知识体系, 培养 学生探索新知识的兴趣和进取精神。在教学过程中, 坚持 以学生为主体、以教师为主导, 引导和激发学生主动思考 问题独立解决问题, 培养学生探索新知识的兴趣和进取精 神, 明显提高教学目标达成度。

教学实施中将课程的理论知识部分采取专题化的方 式展开教学, 针对各专题, 设计的具体专题与提炼的问题 如下:

(1) 网络管理功能与标准化内容: 针对我校实际网 络中所应用到华为、DLink等多厂商设备的情况, 请学生 提出解决方案实现统一的网络管理。

（2）业务量控制技术：以实际城市拥堵为例，引导 学生思考从业务量控制角度处理解决网络拥堵问题的方 法。

（3）路由选择技术: 结合与生活紧密相关的快递投 送业务, 引导学生思考如何设计最优、最快投送数据包的 方法。

(4) 网络自愈技术: 针对网络自愈的需求, 引导学 生探讨解决方案。

(5) 网络信息安全技术: 针对具体的信息安全问题, 引导学生提出解决思路。

专题化的教学探索, 避免了学生胡子眉毛一把抓的困 惑, 面对庞杂的网络管理的知识, 专题化的教学从一开始 就让学生对将要学习的内容有个较为清晰的认识, 并在认 知上让学生感到理论难度降低了, 能够在一定程度上减轻 他们的畏难情绪, 提升学习信心。

\section{4. 基于PoPBL教学实践案例分析}

“通信网体系结构” 这部分的内容是通信网管理的 一个基础, 这部分内容在先修课程如《计算机网络》等课 程中是学习过的, 但通过课前调查发现, 很多学生掌握得 并不好, 因此计划用一个学时的时间, 基于PoPBL教学模 式展开这部分内容的教学。

课堂教学中主要以学生课堂讨论小组的方式进行, 学 生在教师的引导和启发下, 将教学内容设计成若干问题, 学生通过积极的讨论学习获取相关知识和信息。通过小组 讨论, 学生间不同思想火花的撞击, 有疑问的学生会及时 发现问题症结。这一过程使小组内的所有学生都有所收获 - —遇到困难的学生解决了疑问; 提供帮助的学生也通过 解释疑问理清了思路, 加深了对相关内容的理解: 养成学 生思考问题、解决问题的技能和自主学习的能力, 为终身 学习打下良好基础。

教师组织实施过程中, 需注意: (1)可以是教师先抛出 问题, 让学生带着问题课外自学后, 再来进行课堂讨论解 疑; 也可以是学生先自学后提出系列问题及解答方案, 教 师引导着组织学生进行真假问题的讨论辨析, 对小组讨论 后仍有不明确的部分真问题进行探讨并解疑。(2)课堂讨论 小组建议同学期同课程保持相对固定。(3)教师课前要提出 详细的问题讨论提纲, 包括学生可能提出的一些问题的预 设、相关解答方案、具体的讨论要求等。(4)课堂中组织讨 论小组进行自由讨论, 每次讨论时间要控制在10分钟左右, 教师要密切关注学生的讨论过程, 及时纠正一些偏离讨论 方向和主题的争论。(5)学生讨论结束后, 各小组代表就小 组讨论结果及感想发言 (要求有书面材料, 学生互评结束 后上交), 每一小组3 5分钟左右。(6)最后教师进行讨论 小结, 在小结讲授中有所侧重, 注意对讨论中出现的共性 问题和个性问题的统筹指导。

具体到本次课堂教学, 首先以 “在特定的应用背景和 应用需求下, 设计某军事通信网的体系结构” 问题为切入, 通过学生们的讨论与发言加深同学们对体系结构这一概 念以及分层设计思想的理解。这一问题将抽象的概念与方 法放置在需要解决的具体情境下, 能够让学生在抽象的理 论知识与具象生产实践之间搭起桥梁, 增强学生对所学内 容的兴趣, 在一定程度上提高学生对自己解决问题能力的 信心。接下来结合教师预设的问题进行讨论交流, 要求每 个小组代表发言后, 其它两个小组至少各提出 2 个不同的 问题，由发言小组的不同学生回答。提问结束后由发言小 组的未发言过的学生对讨论的要点与知识点进行归纳小 结。本次课借助多媒体和板书, 鼓励学生对于要交流发言 的问题制作课件, 并纳入评价方案。

采用PoPBL教学法时, 宜结合动态评价方案。具体到 本次课程, 评价方案是: 教师给小组打分, 小组以教师给 出的团队成绩为基准, 开展学生间的相互评价, 根据每一 学生实际完成的任务成效决定成绩。同时教师要指导学生 做好并保存相关学习记录, 对自己和他人的学习活动作出 正确评价, 培养学生良好的归因品质。教师还要帮助学生 从自我评价中进行总结、反思, 不断改进学生的学习方法, 提高学生的元认知水平 [5]。 


\section{5. 基于PoPBL教学的几点思考}

PoPBL教学法关注的是学生参与教学活动的主动性、 独立性如何充分发挥, 如何取得更好的教学效果的问题。 通过在通信网管理课程的教学实践, 我们感觉到想达到好 的教学效果, 具体实施该法教学过程中应注意以下问题:

(1) 项目要精选。项目的选择首先要能够引起学生 的学习兴趣, 如果不能达到这个目的, 则无从调动学生的 积极性, 也就失去了 PoPBL模式的基础。为此, 我们选取 的项目主要是以学生天天接触的校园网为基础。从校园网 的实际应用中, 设定场景, 引导学生思考。其次, 项目的 选择应该和所要讨论的知识点契合, 即能够引导学生针对 性的思考, 从而能够深入掌握人才培养方案中要求的知识 点内容。

(2) 问题要提炼。PoPBL是以问题为牵引, 引导学生 的独立思考。因此, 问题必须要明确有针对性, 如果问题 太大太发散, 学生往往不知从何处着手, 问题提炼应该是 实实在在等待学生解决的具体问题, 这样才能充分牵引学 生思考。

(3) 强调以学生的主动学习为主。教师应当注意调 动学生学习的主动性, 帮助学生树立起对自己学习任务的 责任感, 引导学生独立思考、分析并质疑, 鼓励学生发表 自己的见解。

(4) PoPBL教学模式对教师提出了高要求。PoPBL教 学法对教师的知识面、教学水平和工程实践经验提出了较 传统教学方法更高的要求, 在具体实施过程中, 可能由于 实践经验不足, 导致案例或问题缺乏新鲜感, 与实践脱节。 或者由于课时和教学任务的限制, 对典型案例的剖析不够 深入, 将案例教学等同举例说明, 这些将严重影响到预期 的教学效果。因此, 教师必须想办法获得充分的理论知识 和实践经验 [3]。

\section{4. 实验教学方法改革}

本门课程的实验教学为 20 学时, 在课程教学中占比较 重, 同时, 实验教学对于我们的教学目标之一 “提升学生 在网络管理方面的技能” 来说非常重要。因此在实验教学 中我们也想了很多办法来提升教学效果。

\section{1 . 实验教学基本方案}

实验教学中, 抓住网络管理功能与网络管理技术这两 个重要内容, 以知识验证、知识综合为原则设计实验内容, 具体实验教学内容与学时分配如表 2 所示。

表2 实验教学内容与学时分配。

\begin{tabular}{lll}
\hline 序号 & 教学内容 & 实践教学学时 \\
\hline 1 & Windows平台网络测试工具实验 & 2 \\
2 & PackerTracer组网实验 & 6 \\
3 & Web服务和FTP服务实验 & 2 \\
4 & 协议分析软件的使用 & 2 \\
5 & SNMP MIB Browser网管软件使用 & 2 \\
6 & SNMPc 网管软件的使用 & 4 \\
7 & NetInsight 网管软件的使用 & 2 \\
总计 & & 20 \\
\hline
\end{tabular}

具体实施过程中, 我们在学习和理解CDI0教育理念的 基础上, 基于CDIO工程教育模式, 从各实验项目的实施过 程、环节的设置以及考核形式等三方面进行改革。

\subsection{CDI0工程教学模式}

CDI0是构思（Conceive）、设计（Design）、实施 (Implement)、运行 (Operate) 的缩写, 它是麻省理工 学院联合了几所瑞典大学, 从 2000 年开始, 经过长达 4 年 的跨国研究和探索后提出的一个工程教育理念。该理念是 “做中学” 与 “基于项目教育和学习” (Project Based Education and Learning) 的集中概括和抽象表达 [7]。应 该说, CDI0是一种在 “建构主义” 基础上发展起来的工程 教育理念 $[4]$ 。

CDI0工程教育最重要的特点是从 “培养什么人” 以及 “怎样培养人” 这两个根本问题出发来探索工程教育问题。 它的核心内容包括一个愿景、一个教学大纲以及 12 条标准。 它在 “CDI0教学大纲”、“一体化的培养思想”、“以前 两个方面为核心的系统化改革方法” 这三个方面对我国 工程教育改革具有极其重要的参考意义 [7]。

“完整性” 和 “详细性” 是CDIO教学大纲最突出的 贡献。在具体条文上, 它将各种能力要求逐级分解, 直到 可以执行、可以观察的程度。例如, “工程推理和解决问 题的能力”大纲可进一步分解为五个子项; 而每个子项下 可再进一步分解, 直到可以实施、观察的细目, 如 “发现 问题和表述问题” 这一子项下, 可进一步分解为四个细目 $[7]$ 。如图1所示。这样逐级分解的好处是使得教学大纲更 具可操作性。

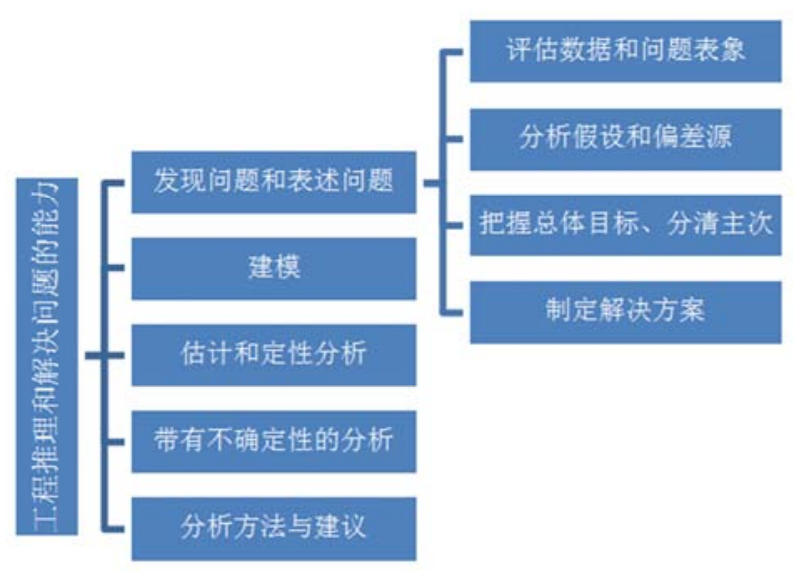

图1 CDI0教学大纲逐级分解示例。

目前，阐述CDI0核心内容的相关文献非常多，本文不 再赘述。从CDIO教学大纲逐级分解示例中可见, 采用CDIO 工程教育理念, “培养学生分析问题和解决问题的能力” 不再是空洞的口号, 而是可以通过课程的具体教学环节来 实现的具体目标 $[7]$ 。

\section{3. 基于 $\mathrm{CDI} 0$ 模式的课程实验教学}

在学习和理解CDI0教育理念的基础上, 基于CDI0工程 教育模式, 从各实验项目的实施过程、环节的设置以及考 核形式等三方面进行改革。 
在实施方式上, 我们从以往的实物搭建平台进行相关 实验转变为以国内外著名网络管理系统和工具 (具体为: PackerTracer、SNMP MIB Browser、SNMPc、NetInsight 等软件) 为平台, 通过实践教学使学生掌握典型网管系统 及常用网管工具的使用方法, 学会使用它们分析网络管理 中常见的问题, 并尝试解决问题, 从而使学生在从事通信 网管理相关工作时能够具备发现问题、分析问题、进而解 决问题的能力。实验教学中, 让学生通过对典型案例设计 开发的学习, 熟悉使用常用网管工具解决实际问题的方法, 掌握一般网管系统的设计开发流程。以期构建起通信网管 理的一般方法与具体解决方案的知识体系。以常用网管系 统和工具为平台的仿真实验实施方式只需要具备计算机 即可实施。同时, 这样的实施方式还具备灵活易修改、不 怕出错等优点, 学生可以根据自己的研究兴趣或者实际工 程需要随意修改, 而在计算机上的仿真实验与在实物上操 作的方法、规程等都是一致的, 具备极高的可移植性。与 传统的实物搭建平台的实施方式相比, 仿真实验实施方式 在灵活性、试错性、提高兴趣、培养网管技能等方面表现 出了非常突出的优点。

在教学环节的设置方面, 尝试将实验教学分为 “课程 设计、课程实验、综合实验”三个环节, 它们的内容相辅 相成。教师首先要结合理论教学知识点设计出若干套设计 课题, 提前布置给学生, 以便学生在理论学习中能够围绕 设计课题来展开, 更有目的性地学习理论知识, 更好地完 成设计课题。在实践中, 学生的综合运用所学知识的能力 和团队分工协作能力都能够得到有效的锻炼。课程设计的 课题可以根据课程的具体的知识点进行划分, 进一步分解 为多个课程实验项目。在这个环节可以根据不同组的学生 的水平差异因材施教, 对于水平较高的分组, 课程实验项 目的划分可以在教师的指导下由学生完成, 对于水平较弱 的分组, 则可以将主要精力放在实验项目的完成上。该环 节的设置不仅可以锻炼学生分析问题的能力, 也可以锻炼 学生分解问题的能力。综合实验环节则要求学生根据实际 网管工程需求, 自行完成需求分析、方案设计、可行性论 证等工作, 该环节设置的目的是为了更好地培养和锻炼学 生的工程实践能力。

由于本课程的实验教学学时占比很重, 因此, 实验课 程的成绩对于课程总成绩也起到至关重要的作用。在实践 课程的考核形式上, 传统的单纯以实验报告打分的形式具 有许多弊端, 因此必须进行改革。通过权衡利弊, 我们采 用了实验报告和综合考核并重的方式来进行实验环节的 考核。采取从实验题库中随机抽取题目, 现场完成, 逐人 通过的方式进行考核, 以完成题目的时间与质量来确定最 终的实验成绩。新的考核方式既 “重过程”也 “重结果” 力求让学生通过实践环节既掌握了知识, 也培养、锻炼了 分析问题解决问题的能力 [4]。

\section{5. 课程考核方式改革}

不论采取哪种教学模式、教学方法展开教学, 都需要 合适的考核方式配合以增进教学效果。下面对课程的考核 方法改革进行小结。
实际授课中, 不少学生养成了被动听讲的习惯, 缺乏 独立思考能力, 有些学生讨论时则很少发言, 学习缺乏主 动性, 不愿意利用业余时间去查询资料, 这样很难取得预 期的教学效果。为了进一步引导学生独立思考、分析并质 疑, 鼓励学生发表自己的见解, 在具体实施各种创新教法 的过程中, 还需要摸索出一套合理的奖惩制度, 鼓励学生 独立完成, 积极配合。为此, 我们在优化考核方式上也做 了探索。将学生学习成绩优劣的评价与理论学习水平、上 机实验与个体综合素质及创新能力三者相挂钩, 从理论考 核、上机操作和作业等多方面综合评测。采用 “ $30+60$ $+10 ”$ 模式, 即理论考试 30 分, 实验成绩 60 分, 平时成绩 10 分。其中实验成绩 60 分中，平时成绩（10个实验每个 5 分）共计50分，综合考核10分。

考核方式方法创新不仅体现在最终考试成绩占比 分配上的变化, 更体现在实践教学考核方式上的改革。 前一节已详细描述了在实验教学考核环节上具体的措 施与手段, 此处不再赘述。考核方式的改革优化对于提 升教学效果起到了显著的 “催化剂” 的作用。

\section{6. 现代化教学手段辅助教学}

如何提高教学效果, 使学生喜欢这门课程、学好这门 课程是改革成败的关键。因此, 我们采用多种现代化教学 手段, 在通信网管理课程教学中起到了很好的作用。

首先, 在教学中, 我们开发研制了计算机辅助教学课 件。教学课件以教材为蓝本, 含简明、清晰的授课讲义、 例题演示等内容, 既有课本内容的直接再现, 又增加了很 多有助于讲解理论的表现手段。课件以计算机为载体, 既 可用于课堂教学, 又可通过网上课程共享平台的方式, 供 学生进行自学和课后复习使用。

其次, 在授课过程中, 基于大专班学生的实际情况 (无 法利用校园网的SPOC教学平台, 但每位学生都有智能手 机), 充分利用了互联网, 建立了班级学习微信群。微信 群的主要功能有:

（1）作业批改后, 及时在群里发布作业完成情况, 对作业质量高的学生进行表扬, 对存在的问题进行讲评。

（2）对学生英语水平较差的状况，每天发布一条通 信网管理中涉及的专业词汇的英文缩写, 要求学生学习并 牢记其英文全称并掌握其中文解释。

(3) 学生有了问题可以及时与教师以及其他学生互 动。

(4) 平时的作业、实验报告得分等均及时通过网络 平台发布, 既让学生对自己的学习状况有比较清晰的了解, 能够在一定程度上起到激励的作用, 又能避免学生对课程 总成绩的质疑。

\section{7. 结论}

由于在教学过程中注重由传统的 “注入式知识传授” 转变为 “研究式素质教育” ; 授课方式由 “连续型细节式 授课” 转变为 “启发式专题授课” ; 教学形式由 “单一的 课堂教学” 转变为 “多形式的互动交流”。课程实施过程 中, 以任务驱动、问题引导为核心, 采用启发式和研讨式 
的教学方法, 抓住 “设疑”、“质疑”、“小结”、“指 定型实验” 和 “分组实验” 等多种有效的形式开展教学, 让学生通过具体的问题解决过程和类比理解掌握知识的 内涵, 讲解时点明问题的思路, 突出重点, 以点带面, 以 缓解教学内容多学时少的矛盾。教学实践表明, 学生对课 程的学习兴趣、对知识点的掌握、对完成实验的信心以及 最终的考试成绩均比以往有明显改善和提高。

虽然我们在理论教学、实验教学以及考核方式等方面 都做了比较多的改革尝试, 教学效果也有明显的改善, 但 该门课程上升的空间仍然很大, 如能在以下两个方面进一 步改革, 相信对改善课程的教学效果将有更大的帮助:

(1) 针对大专班学生学习基础、学习能力等因素差 距较大的客观事实, 如能在分班前先进行简单的水平与能 力测试, 分层次教学将会取得更好的教学效果。实验教学 中我们已经做了一定的尝试, 实验内容分层次, 对于基础 较差的同学要求完成最基本的题目即可, 而对于基础较好 的同学则提出更高的要求。但在理论教学中, 对于一个班 的同学分化较严重的情况还没有很好的对策。

(2) 在教学中发现, 大部分学生起初对待学习的态 度并不端正, 觉得自己无论学好学坏, 肯定能顺利通过考 试, 顺利毕业。但当你对他们严格要求, 对他们的错误的 言行能进行严厉地批评时, 他们也能做出可喜的改变。因 此建议对于大专班学生采取末位淘汰制, 从一入学始就严 格要求。

\section{参考文献}

[1] 郭军编著. 网络管理 (第3 版). 北京邮电大学出版社. 2008. 01。
[2] 张国敏, 陈鸣等. 面向工程实践的网络管理课程教学探索. 计算机教育. 2011, 19:95-97。

[3] 郑翔, 童玮等, PoPBL教学模式在通信网管理课程教学中的 实践与思考. 新时代教育教学. 2014, 10:34-35。

[4] 蒋海霞, 周星宇等. 基于CDI0模式的通信网管理课程实验教 学的探索与实践. 教育教学论坛. 2016, 12:125-126。

[5] 蒋海霞, 张文强等. PBL模式在工科课堂教学中的探索与实 践. 教学探索与教学研究, 2014, 65:19-21。

[6] 高胜利, 赵方方等. PBL教学模式与高素质创新人才的培养. 实验室研究与探索. 2007, 26(5):83-86。

[7] 百度百科. CDI0. http://baike. baidu. com/... 。

[8] 顾佩华, 陆小华. 基于CDI0工程教育改革的探索与实践. http://www. edu.cn/jxyj_10154/20100706/t20100706_49 3393. shtml。

[9] 康叶钦. 在线教育的后 “MOOC时代” - SPOC解析. 清华大学 教育研究. 2014, 39 (1) :85-93。

[10] 陈卫卫, 李清, 李志刚等. 基于概念图和BOPPPS模型的教学 研究与实践. 计算机教育. 2015, 6:61-65。

[11] 黄金丹. 基于PBL的高校工科人才培养机制研究 [D]. 杭州: 浙江大学, 2008。 